hep-th/0009144

ITP-UH-15/00

\title{
On the Integrability of Covariant Field Theory for Open $\mathrm{N}=2$ Strings
}

\author{
Olaf Lechtenfeld and Alexander D. Popov* \\ Institut für Theoretische Physik \\ Universität Hannover \\ Appelstraße 2, 30167 Hannover, Germany \\ Email: lechtenf, popov@itp.uni-hannover.de
}

\begin{abstract}
We discuss the integrability of the Berkovits-Siegel open string field equations and derive an infinite set of their non-local (solution-generating) symmetries. The string field equations are embedded in an infinite system of overdetermined equations (BS hierarchy) associated with hidden string symmetries. The latter enforce the vanishing of most scattering amplitudes for the open $N=2$ string.
\end{abstract}

\footnotetext{
* On leave from Bogoliubov Laboratory of Theoretical Physics, JINR, Dubna, Russia
} 


\section{Introduction}

Although string field theory has not yet led to a non-perturbative and background-independent formulation of string theory, it is a useful tool to illuminate the relation between string and particle dynamics. Besides popular issues like tachyon condensation and non-commutative products, string field theory teaches us how to extend gauge theory and gravity to the string realm, by including the dynamics of an infinite tower of massive excitations.

Such an extension would appear to be rather trivial for self-dual field theories in $2+2$ dimensions: The corresponding critical string theory has $N=2$ world-sheet supersymmetry but lacks any massive physical excitations! Its massless ground state describes gluons and gravitons of same helicity only, but in a light-cone gauge-fixed formulation [1]. Yet, this system shows $S O(2,2)$ Lorentz covariance in the sense that its field equations, the self-duality conditions

$$
R_{\alpha \beta}=0 \quad F_{\alpha \beta}=0,
$$

can be expressed covariantly while there is no Lorentz-invariant action for them alone. This state of affairs makes it awkward to extend the theory off-shell, but it can be remedied by including in the Lagrangian density $\mathcal{L}$ the other helicity degree of freedom as dynamical Lagrange multiplier [2],

$$
\mathcal{L}=\operatorname{Tr} B^{\alpha \beta} F_{\alpha \beta} \quad \mathcal{L}=\operatorname{Tr} \rho^{\alpha \beta} R_{\alpha \beta}
$$

(all fields are Lie algebra-valued). One may wonder if 'integrating in' such multiplier fields is also possible on the string theory side and may lead to a Lorentz-covariant $N=2$ string field theory.

Indeed, Berkovits and Siegel [3] have proposed such a covariant string field theory for the open $N=2$ string, based on an earlier non-covariant version of Berkovits 跑. Their action differs from the standard Chern-Simons-like action [5] in two ways. First, it contains not a single but two string fields, $\Phi$ and $\tilde{\Phi}$, where $\Phi$ contains the prepotential for the self-dual gauge field $A_{\alpha \dot{\alpha}}$, and $\tilde{\Phi}$ functions as a Lagrange multiplier string field multiplying the $\Phi$ equation of motion. Second, in analogy with self-dual Yang-Mills [2], $\Phi$ and $\tilde{\Phi}$ are interpreted as a prepotential for a covariant string field $A_{\alpha}$ and a component of a Lagrange multiplier string field $B_{\alpha \beta}$ for the self-dual field strength $F_{\alpha \beta}(A)$, respectively. Note that these covariant string fields carry space-time indices, but transform only under one of the two $S L(2, \mathbb{R})$ pieces of the $S O(2,2)$ Lorentz group, because the space-time derivative $\partial_{\alpha \dot{\alpha}}$ of the field theory gets replaced by the action of two BRST operators $G_{\alpha}$ for the $N=2$ string, obtained from a "small" $N=4$ superconformal algebra after twisting. The covariant $N=2$ string field action

$$
S=\int \operatorname{Tr} B^{\alpha \beta} * F_{\alpha \beta}(A)
$$

is formulated using Witten's string field product and integral [5] (all fields are string fields now). In the non-covariant Yang or Leznov gauges, the string field equations of motion based on Eq.(1.3),

$$
0=F_{\alpha \beta}=\left\{\nabla_{\alpha}, \nabla_{\beta}\right\} \quad \text { with } \quad \nabla_{\alpha}=G_{\alpha}+A_{\alpha},
$$

reduce to (Chern-Simons-like) Yang-type [6] or (quadratic) Leznov-type [7] equations for the string field $\Phi$ (viz. $\partial_{\alpha \dot{\alpha}} \rightarrow G_{\alpha}$ ), but possess residual gauge invariances due to the non-empty kernels of $G_{\alpha}$. Also, in contrast to the standard NSR $N=1$ string field theory [5], the action (1.3) and

\footnotetext{
${ }^{1}$ We use $4 D$ real Weyl spinor notation for space-time indices, $\alpha \leftrightarrow S L(2, \mathbb{R})$ and $\dot{\alpha} \leftrightarrow S L(2, \mathbb{R})^{\prime}$, with $\alpha=0,1$ and $\dot{\alpha}=\dot{0}, \dot{1}$.
} 
its gauge-fixed variants do not suffer from tree-level divergences due to colliding picture-raising operators [8]. This problem, however, can be avoided also in the NSR formulation by properly distributing left- and right-moving picture-raisers [9, 10].

In this paper, we generalize to the string level the twistor construction [11, 12] of self-dual gauge fields. Namely, using an extra parameter $\zeta \in \mathbb{C} P^{1}$, we introduce a free string field encoding all information about the solutions of the Berkovits-Siegel (BS) string field equations (1.4). The two operators $G_{\alpha}$ are connected with target space-time coordinates. To the (other) coordinates on the moduli space of self-dual gauge fields we correspond new BRST-like operators. A hierarchy of string field equations emerges, containing the BS string field equations (1.4) as well as equations governing their symmetries. In this way, the hidden non-local symmetries of the self-dual Yang-Mills (SDYM) equations get elevated to the string field theory level.

\section{Covariant field theory for open $\mathrm{N}=2$ strings}

From the world-sheet point of view, critical open $N=2$ strings in flat Kleinian space $\mathbb{R}^{2,2}$ are a theory of $N=2$ supergravity on a $1+1$ dimensional (pseudo) Riemann surface with boundaries, coupled to two chiral $N=2$ massless matter multiplets $(X, \psi)$. The latter's components are complex scalars (the four string coordinates) and $S O(1,1)$ Dirac spinors (their four NSR partners). The world-sheet action enjoys local $N=2$ super coordinate and Weyl invariance on the world-sheet, as well as global $U(1,1)$ target space-time symmetry. In the superconformal gauge, the associated left-moving constraints

$$
\begin{aligned}
& T=\partial_{z} X^{\alpha \dot{\beta}} \partial_{z} X_{\alpha \dot{\beta}}+\psi^{\dot{\alpha} \ddot{\beta}} \partial_{z} \psi_{\dot{\alpha} \ddot{\beta}} \quad, \\
& G^{0 \ddot{1}}=\psi^{\ddot{\gamma} \ddot{1}} \partial_{z} X_{\dot{\gamma}}^{0} \quad, \quad G^{1 \ddot{0}}=\psi^{\ddot{\gamma} \ddot{0}} \partial_{z} X_{\dot{\gamma}}^{1} \text {, } \\
& J^{\dddot{0} \ddot{1}}=\psi^{\dot{\gamma} \ddot{0}} \psi_{\dot{\gamma}} \ddot{1}
\end{aligned}
$$

form a $c=6 N=2$ superconformal algebra, where $\ddot{\alpha} \leftrightarrow S L(2, \mathbb{R})^{\prime \prime}$ is the world-sheet internal index, $\ddot{\alpha}=\ddot{0}, \ddot{1}$ associated with the local $U(1) \mathrm{R}$ symmetry. In the NSR formulation, the standard quantization procedure proceeds by introducing ghost systems, extending the contraints to a $c=0$ algebra, writing the BRST operator, and computing its (relative) cohomology for various ghost and picture numbers.

Alternatively, Berkovits and Vafa [13] developed a ghost-free formulation of the $N=2$ string by making use of the extension of (2.1) to the "small" $N=4$ superconformal algebra

$$
\begin{gathered}
T=\partial_{z} X^{\alpha \dot{\beta}} \partial_{z} X_{\alpha \dot{\beta}}+\psi^{\dot{\alpha} \ddot{\beta}} \partial_{z} \psi_{\dot{\alpha} \ddot{\beta}}, \\
G^{\alpha \ddot{\beta}}=\psi^{\dot{\gamma} \ddot{\beta}} \partial_{z} X_{\dot{\gamma}}^{\alpha}, \\
J^{\ddot{\alpha} \ddot{\beta}}=\psi^{\ddot{\gamma} \ddot{\alpha}} \psi_{\dot{\gamma}}^{\ddot{\beta}} .
\end{gathered}
$$

After twisting this algebra, $G^{\alpha \ddot{0}}$ become two fermionic spin-one generators which subsequently serve as BRST-like currents. In this paper, we denote them by $G_{\alpha}:=\epsilon_{\alpha \beta} G^{\beta \ddot{0}}$.

Following Berkovits and Siegel [3], we introduce two Lie algebra-valued fermionic string fields $A_{\alpha}[X, \psi]$, where the arguments $X^{\alpha \dot{\alpha}}(\sigma)$ and $\psi^{\ddot{\alpha} \ddot{\alpha}}(\sigma)$ with $\sigma \in[0, \pi]$ denote the open string configuration. (Note that the Lagrange multiplier string fields, mentioned in the Introduction, reside in 
another multiplet, $B_{\alpha \beta}[X, \psi]$.) Although we suppress it in our notation, string fields are always multiplied using Witten's star product [5]. The three BS string equations of motion (1.4) read[2]

$$
G_{1} A_{1}+A_{1}^{2}=0, \quad G_{0} A_{1}+G_{1} A_{0}+A_{0} A_{1}+A_{1} A_{0}=0, \quad G_{0} A_{0}+A_{0}^{2}=0,
$$

and admit the following two gauges for $A_{\alpha}$ :

$$
\begin{aligned}
& A_{0}=0 \text { and } A_{1}=e^{-\Phi} G_{1} e^{\Phi}, \\
& A_{0}=0 \text { and } A_{1}=G_{0} \Psi \text {, }
\end{aligned}
$$

where $\Phi$ and $\Psi$ are bosonic string fields. The reality conditions on these string fields are [3]:

$$
\Phi^{\dagger}[X(\sigma), \psi(\sigma)]=\Phi[X(\pi-\sigma), \psi(\pi-\sigma)] \quad, \quad \Psi^{\dagger}[X(\sigma), \psi(\sigma)]=\Psi[X(\pi-\sigma), \psi(\pi-\sigma)] .
$$

The gauges (2.4) and (2.5) are analogues of the Yang [6] and Leznov [7] gauges in the SDYM theory. For the Yang gauge (2.4), Eqs.(2.3) are reduced to

$$
G_{0}\left(e^{-\Phi} G_{1} e^{\Phi}\right)=0
$$

whereas for the Leznov gauge (2.5), they simplify to

$$
G_{1} G_{0} \Psi+G_{0} \Psi G_{0} \Psi=0 .
$$

The Yang-type Eq.(2.7) as well as the Leznov-type Eq.(2.8) are non-linear generalization of the "wave" equation $G_{1} G_{0} \Psi=0$.

The gauge symmetries of Eqs.(2.3), (2.7), and (2.8) are described in [3]. Concretely, the covariant equations (2.3) are invariant under the transformations

$$
A_{\alpha} \mapsto \tilde{A}_{\alpha}=e^{K}\left(G_{\alpha}+A_{\alpha}\right) e^{-K},
$$

where $K$ is arbitrary. For the gauge-fixed equations (2.7) and (2.8), this translates to invariances under

$$
e^{\Phi} \mapsto e^{\tilde{\Phi}}=e^{\Phi} e^{-N} \quad \text { with } \quad N=G_{0} \Xi
$$

and

$$
\delta \Psi=\left(G_{1} \Xi+\left[G_{0} \Xi, \Psi\right]\right)
$$

respectively, where $\Xi$ is an unconstrained string field.

\section{Integrability of the BS string field equations}

In this section we obtain the BS string field equations (2.3) as the compatibility conditions of some linear equations and give a general recipe for solving them. Consider the extended complex plane $\mathbb{C} \cup\{\infty\}=\mathbb{C} P^{1}$ with a coordinate $\zeta$ and introduce on it two coordinate patches,

$$
\bar{H}_{+}^{2}=\{\zeta \in \mathbb{C} \cup\{\infty\}: \operatorname{Im} \zeta \geq 0\} \quad \text { and } \quad \bar{H}_{-}^{2}=\{\zeta \in \mathbb{C} \cup\{\infty\}: \operatorname{Im} \zeta \leq 0\},
$$

with the overlap

$$
S^{1}=\mathbb{R} P^{1}=\bar{H}_{+}^{2} \cap \bar{H}_{-}^{2}=\{\zeta \in \mathbb{C} \cup\{\infty\}: \operatorname{Im} \zeta=0\} .
$$

\footnotetext{
${ }^{2}$ Since the star product is not (graded) commutative, field squares do not vanish.
} 
Using $G_{\alpha}, A_{\alpha}$, and $\zeta$, we define the following linear equation

$$
\left(G_{1}+\zeta G_{0}+A_{1}+\zeta A_{0}\right) \Psi_{+}=0,
$$

where $\Psi_{+}=\exp \left(\Phi_{+}\right)$, and $\Phi_{+}$is a string field depending not only on $X(\sigma)$ and $\psi(\sigma)$ but also on the parameter $\zeta \in \bar{H}_{+}^{2}$. If $A_{\alpha}$ is given, then (3.3) is an equation for the field $\Psi_{+}$. Solutions $\Psi_{+}$of this linear equation exist (and are functionals of $A_{\alpha}$ ) if the equation

$$
\begin{gathered}
\left(G_{1}+\zeta G_{0}+A_{1}+\zeta A_{0}\right)^{2}=0 \Leftrightarrow \\
\left(G_{1} A_{1}+A_{1}^{2}\right)+\zeta\left(G_{1} A_{0}+G_{0} A_{1}+A_{1} A_{0}+A_{0} A_{1}\right)+\zeta^{2}\left(G_{0} A_{0}+A_{0}^{2}\right)=0
\end{gathered}
$$

is satisfied for any $\zeta \in \bar{H}_{+}^{2}$. This statement is equivalent to the three BS equations (2.3). Moreover, Eqs.(2.3) are also the integrability condition for the linear equation

$$
\left(G_{1}+\zeta G_{0}+A_{1}+\zeta A_{0}\right) \Psi_{-}=0,
$$

where $\Psi_{-}=\exp \left(\Phi_{-}\right)$, and $\Phi_{-}[X(\sigma), \psi(\sigma), \zeta]$ is a string field depending also on $\zeta \in \bar{H}_{-}^{2}$.

Two remarks are in order. First, finding $\Psi_{ \pm}$for given $A_{\alpha}$ is a "direct transform" $A_{\alpha} \rightarrow \Psi_{ \pm}$ in the terminology of integrable systems, and finding $A_{\alpha}$ for given $\Psi_{ \pm}$is an "inverse transform" $\Psi_{ \pm} \rightarrow A_{\alpha}$. Second, Eq.(3.4) can be cast in Chern-Simons form

$$
Q_{0} A+A^{2}=0
$$

by introducing

$$
Q_{0}:=G_{1}+\zeta G_{0} \quad \text { and } \quad A:=A_{1}+\zeta A_{0} .
$$

Hence, the BS equations follow from the Chern-Simons-like equation (3.6). Fields killed by the operator $Q_{0}$ will be called chiral.

Notice that the gauges (2.4) and (2.5) are connected with the following respective choices of asymptotic behavior for $\Psi_{ \pm}$(cf. [14)

$$
\begin{array}{ll}
\Psi_{ \pm}=e^{-\Phi}+O(\zeta) & \text { for } \zeta \rightarrow 0, \\
\Psi_{ \pm}=1+\zeta^{-1} \Psi+O\left(\zeta^{-2}\right) & \text { for } \zeta \rightarrow \infty .
\end{array}
$$

Indeed, inserting (3.8) or (3.9) into Eqs.(3.3) and (3.5) yields formula (2.4) or (2.5). Recall that the string fields $\Phi$ in (2.4), $\Psi$ in (2.5), and $\Phi_{ \pm}$in (3.3) and (3.5) carry Chan-Paton factors which are suppressed throughout this paper.

Now let us introduce the string field

$$
\Upsilon_{+-}:=\Psi_{+}^{-1} \Psi_{-}=e^{-\Phi_{+}} e^{\Phi_{-}}
$$

defined on the overlap $\bar{H}_{+}^{2} \cap \bar{H}_{-}^{2}=S^{1}$, so that it also depends on $\zeta \in S^{1}$. From Eqs.(3.3) and (3.5) it follows that

$$
Q_{0} \Upsilon_{+-} \equiv\left(G_{1}+\zeta G_{0}\right) \Upsilon_{+-}=0,
$$

i.e. $\Upsilon_{+-}=: e^{\Phi_{+-}}$is a free chiral string field depending on an extra parameter $\zeta=\cot \frac{\theta}{2}, 0 \leq \theta \leq 2 \pi$. Thus, constructing solutions of the BS equations (2.3) is equivalent to finding a field $\Upsilon_{+-}$satisfying (3.11) and splitting $\Upsilon_{+-}=\Psi_{+}^{-1} \Psi_{-}$. Then $A_{\alpha}$ can be obtained from $\Psi_{ \pm}$by formulae (3.3), (3.5) or directly from (3.8), (3.9) and (2.4), (2.5). Notice that the zero-mode sector of $\Upsilon_{+-}$contains a transition matrix of a holomorphic bundle over the twistor space [11, 12] which encodes all information about self-dual gauge fields. 


\section{Symmetries and a BS hierarchy}

We now analyze Grassmann-type symmetries generalizing the known symmetries [3] $\delta_{\alpha} \Psi=G_{\alpha} \Psi$ of the BS equations (2.3) in the Leznov gauge (2.5). In other words, we investigate infinitesimal deformations $\Psi \mapsto \Psi+\varepsilon \delta \Psi$ of solutions to the Leznov-type Eq.(2.8), where $\varepsilon$ is a constant Grassmann parameter. Clearly, $\delta: \Psi \mapsto \delta \Psi$ deserves to be called an infinitesimal symmetry transformation if with $\Psi$ also $\Psi+\varepsilon \delta \Psi$ satisfies Eq.(2.8) to first order in $\delta \Psi$, from which it follows that

$$
G_{1} G_{0} \delta \Psi+G_{0} \Psi G_{0} \delta \Psi-G_{0} \delta \Psi G_{0} \Psi=0 \text {. }
$$

To find infinitesimal symmetries of Eq.(2.8) means to find solutions $\delta \Psi$ of Eq.4.1) for any given solution $\Psi$ of Eq.(2.8). Analogously one may consider symmetries of Eq.(2.7).

The BS equations (2.3) and their reduced forms (2.7) and (2.8) can be embedded in an infinite system of overdetermined equations, in the sense that every solution to the BS equations can be extended to a simultaneous solution of this infinite system. The latter will be called the BS hierarchy of equations and describes, in particular, the symmetries of the BS equations which we shall derive.

We recall that the zero-mode sector of Eq.(2.8) is precisely the Leznov field equation, describing SDYM in the Leznov gauge [3]. From SDYM theory we know that the solution of the Leznov field equation depends not only on the space-time coordinates $x^{0 \dot{\alpha}}$ and $x^{1 \dot{\alpha}}$ but also on coordinates $x^{2 \dot{\alpha}}, \ldots, x^{2 J \dot{\alpha}}$ on the moduli space of self-dual gauge fields, where $2 J$ is any positive integer or infinity. In fact, this dependence may be considered as a manifest one (see 14 and references therein). When lifting the solution of the Leznov field equation to the Leznov string field $\Psi[X, \psi]$ solving (2.8), it is therefore natural to promote not only the space-time coordinates but also these moduli coordinates to the string level,

$$
X^{m \dot{\alpha}}(\sigma, \tau)=x^{m \dot{\alpha}}+p^{m \dot{\alpha}} \tau+\text { oscillations } \quad \text { for } \quad m=0, \ldots, 2 J
$$

and consider all string fields as fields depending on $\psi^{\dot{\alpha} \ddot{\beta}}(\sigma)$ and $X^{m \dot{\alpha}}(\sigma)$. Effectively, we have extended the range of the space-time $S L(2, \mathbb{R})$ spinor indices $\alpha=0,1$ to $m=0, \ldots, 2 J$. In this sense, the $N=2$ string lives in a $2(2 J+1)$ dimensional target space-time. In the first-quantized approach, one may consider the operator product expansions

$$
X^{m \dot{\alpha}}(z, \bar{z}) X^{n \dot{\beta}}(w, \bar{w}) \sim g^{m \dot{\alpha}, n \dot{\beta}} \ln |z-w|^{2} \quad,
$$

where $g_{m \dot{\alpha}, n \dot{\beta}}$ is a suitable non-degenerate metric on the $2(2 \mathrm{~J}+1)$ dimensional target space-time, which, upon restriction to the Kleinian $4 D$ space-time, reduces to the flat metric $g_{m \dot{\alpha}, n \dot{\beta}}=\epsilon_{m n} \epsilon \dot{\alpha} \dot{\beta}$ with $m, n=0,1$. We shall also use coordinates

$$
X_{m \dot{\alpha}}:=g_{m \dot{\alpha}, n \dot{\beta}} X^{n \dot{\beta}}
$$

Generalizing $G_{\alpha}^{\ddot{\beta}}$, we introduce the operators

$$
G_{m}^{\ddot{\beta}}=\psi^{\dot{\gamma} \ddot{\beta}} \partial_{z} X_{m \dot{\gamma}} .
$$

After twisting one obtains the BRST-like fermionic operators

$$
G_{m}:=G_{m}{ }^{0},
$$


the first two of which, $G_{0}$ and $G_{1}$, are associated with the coordinates $X_{0 \dot{\alpha}}$ and $X_{1 \dot{\alpha}}$. All the operators (4.6) share the properties of $G_{0}$ and $G_{1}$, e.g. $\left\{G_{m}, G_{n}\right\}=0$. Moreover, any two consecutive operators $G_{m}$ and $G_{m+1}$ can be taken instead of $G_{0}$ and $G_{1}$, since the choice of four functions from $\left\{X^{m \dot{\alpha}}\right\}$ as four target coordinates is arbitrary. For this reason, the string field $\Upsilon_{+-}$(depending now on $X^{m \dot{\alpha}}$ ) from (3.10) may be subjected to the conditions (cf.[14])

$$
Q_{m} \Upsilon_{+-}:=\left(G_{m+1}+\zeta G_{m}\right) \Upsilon_{+-}=0 \quad \text { with } m=0, \ldots, 2 J-1 .
$$

String fields annihilated by the operators $Q_{m}$ will be called chiral fields. As before, $\Upsilon_{+-}$is a free chiral bosonic string field with hidden Chan-Paton factors.

Substituting the splitting (3.10) into (4.7) and using an extension to Liouville's theorem, we obtain

$$
\Psi_{+}\left(G_{m+1}+\zeta G_{m}\right) \Psi_{+}^{-1}=\Psi_{-}\left(G_{m+1}+\zeta G_{m}\right) \Psi_{-}^{-1}=A_{m+1}+\zeta \tilde{A}_{m}
$$

where $A_{m+1}$ and $\tilde{A}_{m}$ are some string fields not depending on $\zeta$. Equations (4.8) can be rewritten as linear equations on $\Psi_{ \pm}$,

$$
\left(G_{m+1}+A_{m+1}+\zeta G_{m}+\zeta \tilde{A}_{m}\right) \Psi_{ \pm}=0 .
$$

The compatibility conditions of Eqs.(4.9) are the following equations

$$
\begin{aligned}
0 & =G_{m+1} A_{n+1}+G_{n+1} A_{m+1}+A_{m+1} A_{n+1}+A_{n+1} A_{m+1}, \\
0 & =G_{m} \tilde{A}_{n}+G_{n} \tilde{A}_{m}+\tilde{A}_{m} \tilde{A}_{n}+\tilde{A}_{n} \tilde{A}_{m}, \\
0 & =G_{m} A_{n+1}+G_{n} A_{m+1}+G_{n+1} \tilde{A}_{m}+G_{m+1} \tilde{A}_{n}+\left\{\tilde{A}_{m}, A_{n+1}\right\}+\left\{\tilde{A}_{n}, A_{m+1}\right\},
\end{aligned}
$$

where $m, n=0, \ldots, 2 J-1$. We name Eqs.(4.10) the truncated $B S$ hierarchy equations. For $J \rightarrow \infty$ the full BS hierarchy equations emerge.

Imposing on $\Psi_{ \pm}$the asymptotic conditions (3.8) and (3.9), where $\Phi$ and $\Psi$ depend now on all $X^{m \dot{\alpha}}$, we obtain the gauges

$$
\begin{aligned}
& \tilde{A}_{m}=0 \text { and } \quad A_{m+1}=e^{-\Phi} G_{m+1} e^{\Phi}, \\
& \tilde{A}_{m}=0 \quad \text { and } \quad A_{m+1}=G_{m} \Psi .
\end{aligned}
$$

For the Leznov gauge (4.12) the BS hierarchy $(4.10)$ reduces to the following equations:

$$
G_{m+1} G_{n} \Psi+G_{n+1} G_{m} \Psi+G_{m} \Psi G_{n} \Psi+G_{n} \Psi G_{m} \Psi=0
$$

containing the Leznov-type Eq.(2.8) at $m=n=0$.

The first novel equations beyond (2.8) appear for $m=0, n \geq 1$. Acting by $G_{0}$ on the left yields

$$
G_{1} G_{0}\left(G_{n} \Psi\right)+G_{0} \Psi G_{0}\left(G_{n} \Psi\right)-G_{0}\left(G_{n} \Psi\right) G_{0} \Psi=0 .
$$

Comparing this with (4.1), we discover that

$$
\delta_{n} \Psi=G_{n} \Psi
$$

are solutions of Eqs.(4.1). Moreover, Eq.(4.13) provides a recursion relation,

$$
\delta_{n+1} \Psi \equiv G_{n+1} \Psi=G_{0}^{-1}\left(G_{1} \delta_{n} \Psi+G_{0} \Psi \delta_{n} \Psi+\delta_{n} \Psi G_{0} \Psi\right)
$$


from which we see that the symmetries $(4.15)$ are non-local if $n \neq 0,1$. It is important to note that $G_{0}$ has a non-zero kernel, so that (2.8) or (4.16) for $n=0$ does not imply $G_{1} \Psi$ being $O\left(\Psi^{2}\right)$. Analogously one can describe the symmetries of the Yang-type equation (2.7).

On the linearized level and on mass-shell the string field $\Psi$ reduces to a first-quantized vertex operator [3]. Up to gauge transformations, the only momentum-dependent $U(1)$-neutral vertex operator satisfying the linear "wave" equation $G_{1} G_{0} \Psi=0$ is (see [13])

$$
\Psi=A \exp \left(i k_{0 \dot{\alpha}} x^{0 \dot{\alpha}}+i k_{1 \dot{\alpha}} x^{1 \dot{\alpha}}\right)
$$

where the momenta $k_{0 \dot{\alpha}}$ and $k_{1 \dot{\alpha}}$ satisfy the on-shell condition

$$
k_{00} k_{1 \dot{1}}-k_{0 \dot{1}} k_{1 \dot{0}}=0
$$

Here, $A$ is a multiplier depending on moduli parameters $x^{m \dot{\alpha}}$ with $m \geq 2$ but not on space-time coordinates $x^{0 \dot{\alpha}}$ or $x^{1 \dot{\alpha}}$. Moreover, on the linearized level the action of the operators $G_{0}$ and $G_{1}$ corresponds merely to a multiplication of the vertex operator $\Psi$ by $\psi^{\dot{\alpha} 0} k_{0 \dot{\alpha}}$ and $\psi^{\ddot{\alpha} 0} k_{1 \dot{\alpha}}$, respectively [13]. Thanks to (4.18), this implies the identity

$$
G_{0} \Psi=h(k) G_{1} \Psi
$$

where [13]

$$
h(k):=\frac{k_{0 \dot{0}}}{k_{1 \dot{0}}}=\frac{k_{0 \mathrm{i}}}{k_{1 \dot{1}}} .
$$

Being recursively connected with space-time translations, our symmetries (4.16) can be compared with the hidden symmetries of the open $N=2$ string constructed in [14 from momentum operators $P_{0 \dot{\alpha}}$ plus operators of picture-raising and spectral flow in the first-quantized BRST approach. The linearization of the BS hierarchy in Leznov gauge (4.13) reads

$$
G_{m+1} G_{n} \Psi+G_{n+1} G_{m} \Psi=0 .
$$

Taking $m=0$ or linearizing (4.16) yields

$$
G_{n} \Psi=G_{0}^{-1}\left(G_{1} G_{n-1} \Psi\right)
$$

which, due to (4.19), simplifies to

$$
\delta_{n}^{\operatorname{lin}} \Psi=G_{n} \Psi=h(k)^{-1} G_{n-1} \Psi=h(k)^{-n} G_{0} \Psi=h(k)^{-n} k_{0 \dot{\alpha}} \psi^{\ddot{\alpha} \ddot{0}} \Psi
$$

It follows that Eqs. (4.21) for $m, n \geq 1$ are automatically satisfied. With $\delta_{n}^{\text {lin }}=\psi^{\dot{\alpha} 0} \delta_{n \dot{\alpha}}^{\text {lin }}$, formulae (4.23) are identical to formulae (5.15) of Ref. [14]. Thus, we have described an infinite set of nonlocal symmetries of the covariant field theory for open $N=2$ strings and shown that the linear part (4.23) of these symmetries coincides with the hidden string symmetries [14 found in the BRST approach. Recall that those symmetries are related to flows on the moduli space of self-dual gauge fields.

It has been demonstrated in the topological approach [13] that Eq.44.19) implies the (on-shell) vanishing of any $N=2$ string $n$-point amplitude unless

$$
h\left(k_{1}\right)=h\left(k_{2}\right)=\ldots=h\left(k_{n}\right)
$$


for the external momenta $k_{1}, k_{2}, \ldots, k_{n}$, which is equivalent to all Mandelstam variables being zero. The same result has been obtained in the BRST approach [15, 16] by employing the Ward identities associated with the linearized symmetries (4.23). [l $^{2}$ Invoking $S$-matrix analyticity in the Mandelstam variables, one concludes that only the three-point function escapes the vanishing theorem. If To sum up, we have established the integrable BS hierarchy describing the expected higher-spin symmetries as being responsible for the vanishing results of $N=2$ open string scattering [13].

\section{Acknowledgements}

This work was partially supported by the German Science Foundation (DFG) under the grant LE 838/7-1 and the DFG-RFBR grant no. 99-02-04022. A.D.P. acknowledges support by RFBR under the grant no. 99-01-01076.

\footnotetext{
${ }^{3}$ These facts refer to the closed string but extend to the open case as well [14].

4 There is no conflict with the Coleman-Mandula theorem since the unitarity argument that would kill also the three-point function does not apply in $2+2$ dimensions.
} 


\section{References}

[1] H. Ooguri and C. Vafa, Geometry of N=2 strings, Nucl. Phys. B361 (1991) 469-518; Self-duality and N=2 string magic, Mod. Phys. Lett. A5 (1990) 1389-1398.

[2] G. Chalmers and W. Siegel, The self-dual sector of QCD amplitudes, Phys. Rev. D54 (1996) 7628-7633 hep-th/9606061.

[3] N. Berkovits and W. Siegel, Covariant field theory for self-dual strings, Nucl. Phys. B505 (1997) 139-152 [hep-th/9703154].

[4] N. Berkovits, Super-Poincare invariant superstring field theory, Nucl. Phys. B450 (1995) 90-102, Erratum-ibid. B459 (1996) 439-451 hep-th/9503099.

[5] E. Witten, Noncommutative geometry and string field theory, Nucl. Phys. B268 (1986) 253-322; Interacting field theory of open superstrings, Nucl. Phys. B276 (1986) 291-339.

[6] C.N. Yang, Condition of self-duality for $S U(n)$ gauge fields on Euclidean four-dimensional space, Phys. Rev. Lett. 38 (1977) 1377-1379.

[7] A.N. Leznov, On equivalence of four-dimensional self-duality equations to continual analog of the main chiral field problem, Theor. Math. Phys. 73 (1987) 1233-1237.

[8] C. Wendt, Scattering amplitudes and contact interactions in Witten's superstring field theory, Nucl. Phys. B314 (1989) 209-252.

[9] O. Lechtenfeld and S. Samuel, Gauge-invariant modification of Witten's open superstring, Phys. Lett. B213 (1988) 431-446.

[10] O. Lechtenfeld, From N=2 fermionic strings to superstrings?, Proceedings of the 28th Int. Ahrenshoop Symposium, Wendisch-Rietz, Germany, 1994, DESY preprint 95-027 pp.16 [hep-th/9412242].

[11] R.S. Ward, On self-dual gauge fields, Phys. Lett. A61 (1977) 81-82.

[12] R.S. Ward and R.O. Wells, Twistor geometry and field theory, Cambridge University Press, Cambridge, 1990.

[13] N. Berkovits and C. Vafa, N=4 topological strings, Nucl. Phys. B433 (1995) 123-180 [hep-th/9407190].

[14] T.A. Ivanova and O. Lechtenfeld, Hidden symmetries of the open N=2 string, to appear in Int. J. Mod. Phys. A15 (2000) [hep-th/0007049].

[15] K. Jünemann, O. Lechtenfeld and A.D. Popov, Non-local symmetries of the closed N=2 string, Nucl. Phys. B548 (1999) 449-474 [hep-th/9901164].

[16] K. Jünemann and O. Lechtenfeld, Ward identities and the vanishing theorem for loop amplitudes of the closed N=2 string, Phys. Lett. B474 (2000) 58-66 hep-th/9912016. 